\section{Insulinemia e índice HOMA en niños y adolescentes chilenos}

\author{
SALESA BARJA ${ }^{1}$, PILAR ARNAIZ ${ }^{1}$, ANGÉLICA DOMÍNGUEZ ${ }^{2, a}$, \\ LUIS VILLARROEL ${ }^{2, b}$, BERTA CASSIS ${ }^{2, c}$, OSCAR CASTILLO ${ }^{3, d}$, \\ GIANINA SALOMÓ ${ }^{2, d}$, MARCELO FARÍAS ${ }^{4}$, MANUELA GOYCOOLEA ${ }^{5, e}$, \\ TERESA QUIROGA ${ }^{5}$, FRANCISCO MARDONES ${ }^{2}$
}

\section{Normal plasma insulin and HOMA values among chilean children and adolescents}

Background: Plasma insulin and HOMA (homeostasis model assessment) index, used to determine insulin resistance, do not have local standard values for children and adolescents in Chile. Aim: To establish the normal reference intervals for insulin and HOMA in children and adolescents aged 10-15 years, according to sex and puberal maturation. Material and Methods: A cross-sectional study of 2,153 children and adolescents from Puente Alto County was performed, during 2009 and 2010. Anthropometry and self-report of puberal maturation were assessed. Fasting glucose (hexoquinase) and insulin blood levels (chemiluminiscence), were determined and HOMA index was calculated. Percentile distributions of these variables were calculated. Results: The reference group included only subjects with normal body mass index and fasting blood glucose $(n=1,192)$. Girls had higher insulin and HOMA values than boys $(12.5 \pm 6.0$ and $9.1 \pm 4.9 \mu U / m L(p<0.01)$ and $2.7 \pm 1.4$ and 2.1 $\pm 1,1$ ( $p<0.01)$, respectively). Subjects with Tanner I and II puberal stages had lower insulin and HOMA mean values than subjects with Tanner III and IV $(9.0 \pm 4.3$ and $12.5 \pm 6.2 \mu \mathrm{U} / \mathrm{ml}(p<0.01)$ and $2.0 \pm 1$ and $2.8 \pm 1.4(p<0.01)$, respectively). Conclusions: The 90th percentile of insulin and HOMA distributions according to sex and maturation, was selected as the upper cut-off point to identify individuals with insulin resistance. HOMA cutoff point for Tanner I and II boys was 3.2, for Tanner I and II girls was 4.1, for Tanner III and IV boys was 4.2 and for Tanner III and IV girls was 5.0.

(Rev Med Chile 2011; 139: 1435-1443).

Key words: Adolescent; Child; Insulin resistance.

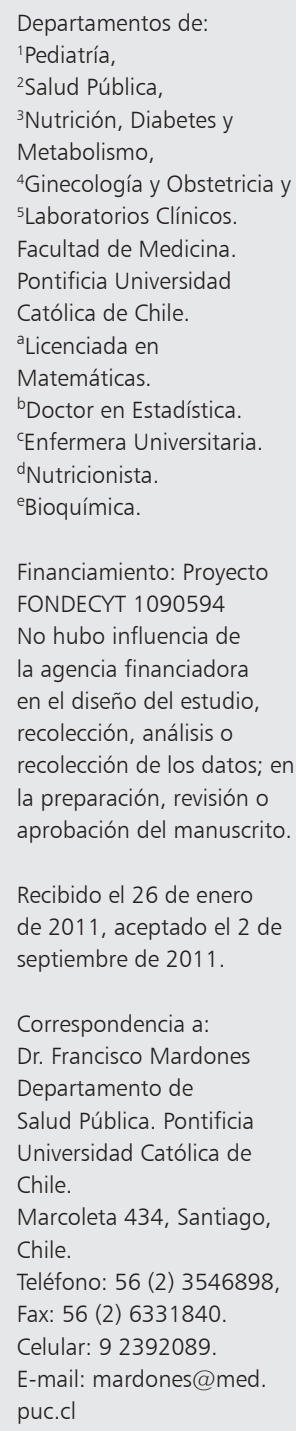

L a resistencia insulínica (RI) constituye una respuesta disminuida de las células blanco a la acción de la insulina, con aumento compensatorio de la secreción de insulina para lograr una respuesta adecuada. Esta secuencia es el principal mecanismo patogénico de la diabetes mellitus tipo $2(\mathrm{DM} 2)^{1}$, así como un posible factor causal común de dislipidemia e hipertensión arterial. Estos componentes del Síndrome Metabólico (SM) descrito por Reaven ${ }^{2}$ que se asocian a obesidad (especialmente abdominal) han aumentado también en población pediátrica, con presentación precoz del SM y mayor riesgo cardiovascular (RCV) futuro ${ }^{3-6}$.

Esta situación epidemiológica ha estimulado la búsqueda de marcadores que identifiquen precozmente a los sujetos con riesgo a desarrollar DM2. En adolescentes se ha demostrado la baja sensibilidad de la glicemia para detectar RI, con mejoría al realizar el test de tolerancia oral a la glucosa (TTOG) ${ }^{7}$. Sin embargo, este es un método poco aplicable para tamizaje, por mayor duración, 
costo y número de muestras sanguíneas. El índice HOMA, construido por Matthews, representa el equilibrio de la relación entre insulinemia y glicemia de ayunas que el organismo realiza para mantener la euglicemia ${ }^{8}$. Tiene buena correlación con métodos de referencia en adultos y niños ${ }^{9,10}$, es simple, de bajo costo, ampliamente utilizado para estudiar la RI y predecir la evolución a DM2 en adultos ${ }^{11}$. El punto de corte superior de HOMA es 2,6 y ha sido validado en adultos jóvenes sanos chilenos ${ }^{12}$.

La medición de la insulinemia tiene limitaciones, como su variabilidad intra e inter-individual y dependencia de la técnica de laboratorio utilizada $^{13}$. Existen además variaciones a lo largo de la vida, con períodos de RI fisiológica durante el embarazo, la vejez y adolescencia ${ }^{14}$. Esto dificulta su interpretación en niños, habiéndose extrapolado en Chile valores de adultos ${ }^{12}$, de niños con sobrepeso $^{15} \mathrm{o}$ de poblaciones extranjeras ${ }^{14}$. Recientemente se publicó una referencia para adultos mayores ${ }^{16}$, pero no la hay para población pediátrica sana.

El objetivo del estudio fue describir los niveles de insulinemia y de HOMA en una muestra de niños y adolescentes chilenos sanos, analizando las variaciones según sexo, edad y maduración puberal, para proponer puntos de corte superior que definan RI.

\section{Material y Método}

Se realizó un estudio transversal en escolares de quinto y sexto básico, durante 2009 y 2010, procedentes de 14 de las 20 escuelas municipalizadas de la comuna de Puente Alto, participantes del proyecto FONDECYT regular (No 1090594), invitados mediante una carta a los apoderados del universo de 3.521 alumnos.

Una enfermera universitaria y una nutricionista realizaron la evaluación en cada escuela en horario matinal, se verificó estado de salud de la semana precedente. Se midió peso y estatura en balanza de palanca con estatímetro, marca Seca ${ }^{\circledR}$, con procedimientos estándar, descalzos, con ropa liviana, descontado el peso promedio de ésta y promediándose tres mediciones. Se calculó el índice talla/edad (T/E) e índice de masa corporal $\left(\mathrm{IMC}=\right.$ Peso en $\mathrm{kg} /$ Talla $^{2}$ en $\left.\mathrm{m}\right)$ expresados en percentiles y puntaje $\mathrm{z}(\mathrm{z}=$ valor real-mediana/1DE), con referencia CDC-NCHS $2000^{17}$. Se catalogó el estado nutricional según percentil de IMC; eutrofia: 5 al 84, sobrepeso: 85 al 94, obesidad: $\geq 95$ y desnutrición: $<5$. Se midió perímetro de cintura (PC) con cinta métrica inextensible, sobre el borde lateral-superior del ilion derecho en línea medio-axilar, al final de una espiración ${ }^{18}$, se promediaron tres mediciones y se usó el valor relativo al promedio de una referencia internacional ${ }^{19}$. Se midieron los pliegues cutáneos tricipital (PTC) y subescapular (PSE) con caliper Harpenden ${ }^{\circledR}$ y técnica estándar ${ }^{18}$, con éstos se calculó la masa grasa (\%MG) con ecuaciones de Slaughter ${ }^{20}$, validadas y aplicadas en niños chilenos ${ }^{21,22}$. En forma privada se solicitó un auto-reporte voluntario del estado puberal con observación de fotos estandarizadas de desarrollo mamario en niñas y genital en hombres, ambas más vello púbico ${ }^{23}$.

A continuación, se obtuvieron muestras de sangre venosa, para determinación de glicemia (método Gluco-quant, Glucosa/ Hexoquinasa, Roche Diagnostics GmbH, Manheim) e insulinemia (inmunoensayo quimio-luminométrico directo, ADVIA Centaur ${ }^{\circledR}$ XP. Bayer HealthCare LLC, Kyowa Medex Co, Japón); éste mide concentraciones de insulina de 0,5-300 $\mu \mathrm{UI} / \mathrm{mL}$ (sensibilidad $0,5 \mu \mathrm{UI} / \mathrm{mL}$ ) con coeficiente de variación de 3,48\% y $6,17 \%$ para concentraciones de $23,51 \mu \mathrm{UI} / \mathrm{mL}$ y $62,49 \mu \mathrm{UI} / \mathrm{mL}$, respectivamente. Se calculó HOMA con la fórmula de Matthews ${ }^{7} ; \mathrm{HOMA}=[($ Glicemia $(\mathrm{mmol} / \mathrm{Lt})$ x Insulinemia $(\mu \mathrm{UI} / \mathrm{mL})) / 22,5]$. Unidades de glicemia: $\mathrm{mmol} / \mathrm{L}=[(\mathrm{mg} / \mathrm{dL}) / 18]$.

Los criterios de inclusión fueron: sujetos sanos, eutróficos y con glicemia de ayunas entre 60 y 99 $\mathrm{mg} / \mathrm{dL}$. Se excluyeron los post-púberes (Tanner V) por constituir una sub-muestra de escaso tamaño $(\mathrm{n}=23)$ y tres pacientes con insulinemia $>60 \mu \mathrm{UI} / \mathrm{mL}$.

Se verificó normalidad en la distribución de variables numéricas con test de Kolmogorov-Smirnov. Su análisis descriptivo se hizo con promedio y desviación estándar (DE) y el de las variables categóricas con número de casos y porcentajes. Se compararon promedios entre dos grupos con test t de Student (muestras independientes), para más de dos grupos se usó análisis de varianza (ANOVA) y test de comparaciones múltiples de Bonferroni, si correspondía. Para asociación lineal simple se usó coeficiente de correlación de Pearson y para determinar variables asociadas en forma conjunta e independiente con insulinemia y HOMA, se usó regresión lineal paso-a-paso (stepwise). Se deter- 
minó la distribución en percentiles de insulinemia y HOMA según sexo y maduración puberal. Se consideró significativo $\mathrm{p} \leq 0,05$. Se utilizó el programa SAS 9.3.

Los padres o sus representantes firmaron un formulario de consentimiento informado y los niños/niñas y adolescentes un formulario de asentimiento informado. El estudio fue aprobado por las comisiones de ética de la Escuela de Medicina, Pontificia Universidad Católica de Chile, y de FONDECYT, CONICYT.

\section{Resultados}

Del total de 3.521 alumnos en el universo, se evaluaron 2.153 niños y adolescentes que presentaron los formularios de consentimiento informado firmados; $31,5 \%$ de las niñas y $44,5 \%$ de los varones no aceptaron participar $(\mathrm{p}<0,0001)$. No hubo diferencias significativas entre aquellos que participaron o no en edad, años de educación materna, edad gestacional o antropometría al nacer. La edad promedio según sexo fue discretamente diferente (Tabla 1), con rango de 10 a 15 años en el grupo total y para cada sexo. El estado nutricional de los 2.153 niños fue: 60,1\% eutróficos, 22,7\% sobrepeso, $14,7 \%$ obesos y $2,5 \%$ bajo peso.

De los 1.285 eutróficos, 1.192 fueron euglicémicos, siendo ésta la población de referencia. Las mujeres fueron 52\% y su estado puberal: 19,7\% $(\mathrm{n}=235)$ tenía Tanner I, 41,4\% $(\mathrm{n}=494)$ Tanner II, 31\% ( $\mathrm{n}=369)$ Tanner III y 7,9\% Tanner IV (n =94). Hubo mayor proporción de Tanner I-II en hombres y de III-IV en mujeres.

Las características generales según sexo revelaron diferencias de baja magnitud, siendo mayores el zT/E, PC, \%MG y obesidad troncal en las mujeres, sin diferencia en zIMC (Tabla 1); éstas tuvieron menor glicemia pero mayor insulinemia y HOMA que los hombres (Figura 1).

La insulinemia y HOMA aumentaron con la maduración puberal (Figura 2). Aunque los sujetos con Tanner I y II presentaron entre sí una diferencia significativa en insulinemia $(8,5 \pm 4,3$ y $9,9 \pm$ $5,1 \mu \mathrm{UI} / \mathrm{mL})$, ésta fue menor que la observada entre ellos y los de Tanner III o IV $(12,9 \pm 6,4$ y $13,3 \pm 6,1 \mu \mathrm{UI} / \mathrm{mL}$ ), sin diferencia entre estos dos últimos. Similar situación ocurrió con HOMA. Considerando lo anterior, se agruparon los dos primeros, quienes al ser comparados con los púberes (Tanner $3+4$ ) tuvieron menor insulinemia y HOMA ( $\mathrm{p}<0,0001)$, persistiendo la diferencia

Tabla 1. Características generales (promedios \pm DS y porcentajes) de 1.192 niños y adolescentes eutróficos y euglicémicos. Comuna de Puente Alto, 2009-2010

\begin{tabular}{|lcccc|}
\hline Medición & Total & $\begin{array}{c}\text { Mujeres } \\
(\mathbf{n = 6 1 9 )}\end{array}$ & $\begin{array}{c}\text { Hombres } \\
(\mathbf{n}=\mathbf{5 7 3})\end{array}$ & $\begin{array}{c}\text { Valor - p } \\
\text { (t Student) }\end{array}$ \\
\hline Edad (años) & $11,4 \pm 1,0$ & $11,4 \pm 1,0$ & $11,5 \pm 0,9$ & 0,03 \\
\hline Peso $(\mathrm{kg})$ & $38,2 \pm 6,6$ & $38,8 \pm 6,7$ & $37,6 \pm 6,5$ & 0,002 \\
\hline Talla $(\mathrm{cm})$ & $145,4 \pm 8,1$ & $145,8 \pm 7,9$ & $144,9 \pm 8,3$ & $\mathrm{~ns}$ \\
\hline zT/E & $-0,16 \pm 0,9$ & $-0,08 \pm 1,0$ & $-0,24 \pm 0,9$ & 0,003 \\
\hline IMC $\left(\mathrm{Kg} / \mathrm{m}^{2}\right)$ & $18,0 \pm 1,7$ & $18,1 \pm 1,9$ & $17,8 \pm 1,6$ & 0,0005 \\
\hline zIMC & $0,06 \pm 0,7$ & $0,09 \pm 0,7$ & $0,03 \pm 0,7$ & $\mathrm{~ns}$ \\
\hline P. Cintura $(\mathrm{cm})$ & $66,9 \pm 5,6$ & $67,5 \pm 5,7$ & $66,3 \pm 5,3$ & 0,0001 \\
\hline P. Cintura $(\%)^{1}$ & $101,5 \pm 8,1$ & $103,0 \pm 8,2$ & $99,8 \pm 7,6$ & $<0,0001$ \\
\hline Masa grasa (\%) & $18,3 \pm 5,7$ & $19,8 \pm 6,1$ & $16,7 \pm 4,8$ & $<0,0001$ \\
\hline Obesidad troncal ${ }^{2}$ & $0,82 \pm 0,2$ & $0,85 \pm 2,3$ & $0,80 \pm 0,2$ & $<0,0001$ \\
\hline Glicemia $(\mathrm{mg} / \mathrm{dL})$ & $89,5 \pm 5,4$ & $88,7 \pm 5,5$ & $90,4 \pm 5,2$ & $<0,0001$ \\
\hline Insulina $(\mu \mathrm{U} / \mathrm{mL})$ & $10,9 \pm 5,8$ & $12,5 \pm 6,1$ & $9,1 \pm 4,9$ & $<0,0001$ \\
\hline HOMA & $2,4 \pm 1,3$ & $2,7 \pm 1,4$ & $2,1 \pm 1,1$ & $<0,0001$ \\
\hline
\end{tabular}

${ }^{1}$ Perímetro de Cintura, expresado como \% del p5019. $2 \%$ Masa Grasa de acuerdo a ecuaciones Slaugther ${ }^{20} .{ }^{3}$ Obesidad troncal: razón de PSE/PTC, en mm. 

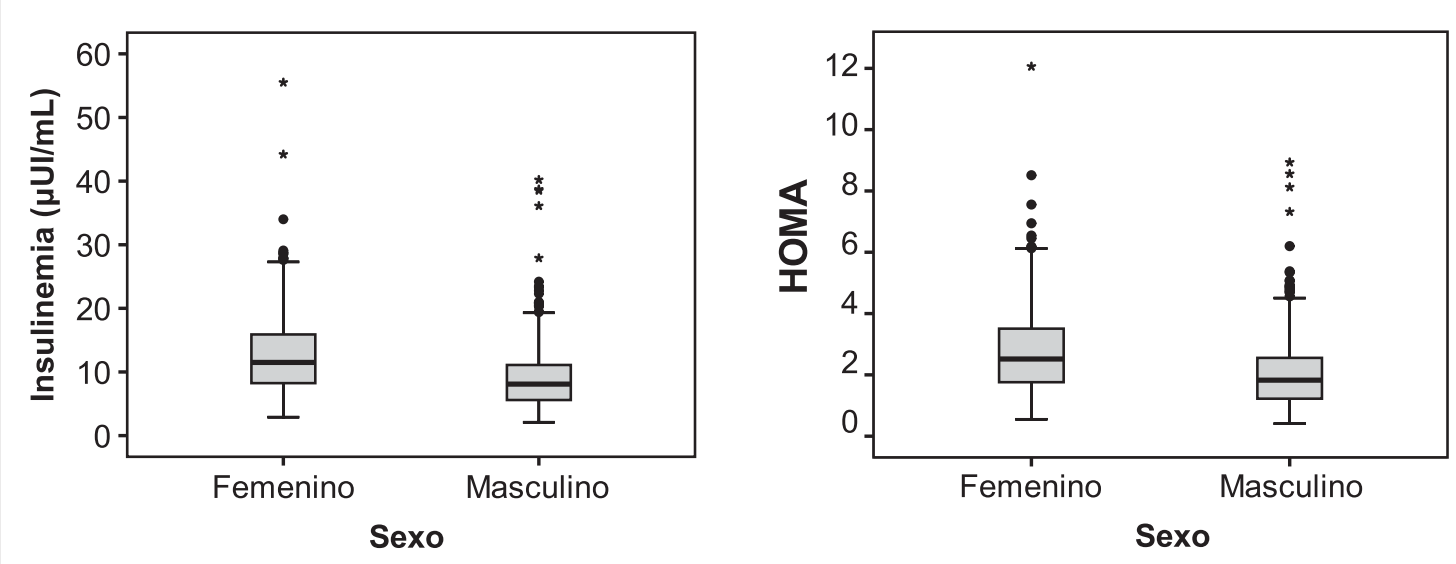

Figura 1. Promedios de insulinemia e índice HOMA en 1.192 niños sanos, eutróficos y euglicémicos, de acuerdo a sexo. Comuna de Puente Alto, 2009-2010. *Para cada comparación: t Student, $p<0,001$.

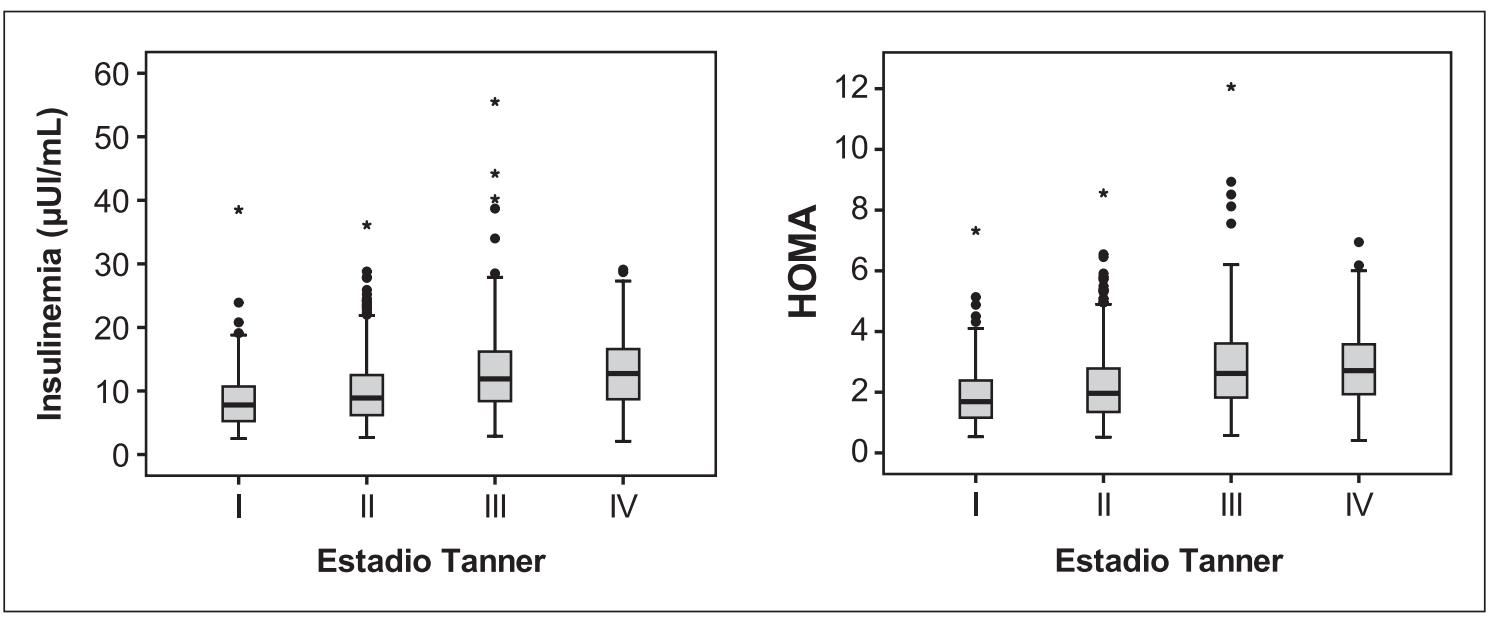

Figura 2. Promedios de insulinemia e índice de HOMA en 1.192 niños sanos, eutróficos y euglicémicos, de acuerdo a etapa de maduración puberal. Comuna de Puente Alto, 2009-2010. Test de Bonferroni: Existe diferencia significativa para Insulinemia y HOMA entre las etapas de Tanner I y II vs III y IV ( $<0,0001)$, sin diferencia entre las etapas III y IV.

según sexo en ambas, independientemente del estado puberal (Tabla 2). Hubo una correlación positiva de la edad con insulinemia $(\mathrm{r}=0,189 \mathrm{p}$ $<0,0001)$ y HOMA $(\mathrm{r}=0,191 \mathrm{p}<0,0001)$, pero no con glicemia $(r=0,05 \mathrm{p}=0,082)$.

Las correlaciones simples de PC con HOMA e insulinemia fueron similares: $r=0,404(p<0,001)$ y r $=0,406(p<0,001)$, respectivamente (Figura $3)$. También fueron similares las correlaciones de HOMA con zIMC $\mathrm{r}=0,327$ ( $\mathrm{p}<0,0001)$ y con $\% \mathrm{MG} \mathrm{r}=0,338(\mathrm{p}<0,0001)$.
La distribución percentilar para insulinemia y HOMA presenta diferentes valores al separar por sexo y grupos de maduración puberal en los diferentes percentiles sobre 75 (Tabla 3 ).

En modelos de regresión lineal, las variables que conjuntamente y por orden de importancia ayudan a explicar la variación de la insulinemia son: $\mathrm{PC}\left(\mathrm{R}^{2}=0,14\right)$, Tanner $\left(\mathrm{R}^{2}=0,06\right)$, sexo $\left(\mathrm{R}^{2}=\right.$ $0,03)$, zIMC $\left(\mathrm{R}^{2}=0,007\right)$ y PSE/PTC $\left(\mathrm{R}^{2}=0,004\right)$, todas $\mathrm{p}<0,001$. El modelo para HOMA seleccionó las mismas variables, con significación similar. 
Tabla 2. Promedios de glicemia, insulinemia e índice de HOMA de acuerdo a sexo y maduración puberal, en 1.192 niños y adolescentes eutróficos y euglicémicos. Comuna de Puente Alto, 2009-2010

\begin{tabular}{|c|c|c|c|c|c|c|}
\hline \multirow[t]{2}{*}{ Medición } & \multicolumn{3}{|c|}{$\begin{array}{l}\text { Prepúberes y púberes iniciales } \\
\text { (Tanner I + II) }\end{array}$} & \multicolumn{3}{|c|}{$\begin{array}{c}\text { Púberes } \\
(\text { Tanner III + IV) }\end{array}$} \\
\hline & $\begin{array}{l}\text { Mujeres } \\
(n=309)\end{array}$ & $\begin{array}{l}\text { Hombres } \\
(n=420)\end{array}$ & $\begin{array}{c}\text { Total } \\
(n=729)\end{array}$ & $\begin{array}{l}\text { Mujeres } \\
(n=310)\end{array}$ & $\begin{array}{l}\text { Hombres } \\
(n=153)\end{array}$ & $\begin{array}{c}\text { Total } \\
(n=463)\end{array}$ \\
\hline Glicemia (mg/dL) & $88,3 \pm 5,8^{*}$ & $90,4 \pm 5,2$ & $89,5 \pm 5,6^{*}$ & $89,0 \pm 5,2^{* *}$ & $90,3 \pm 5,3$ & $89,5 \pm 5,2 *$ \\
\hline Insulinemia ( $\mu \mathrm{U} / \mathrm{mL})$ & $10,6 \pm 5,2^{*}$ & $8,7 \pm 4,5$ & $9,5 \pm 4,9^{*}$ & $14,3 \pm 6,2^{* *}$ & $10,4 \pm 5,8$ & $13,0 \pm 6,4^{*}$ \\
\hline HOMA & $2,3 \pm 1,2^{*}$ & $1,9 \pm 1,0$ & $2,1 \pm 1,1^{*}$ & $3,2 \pm 1,4^{* *}$ & $2,3 \pm 1,3$ & $2,9 \pm 1,4^{*}$ \\
\hline
\end{tabular}

${ }^{*} p<0,0001 .{ }^{* *} p=0,01$.
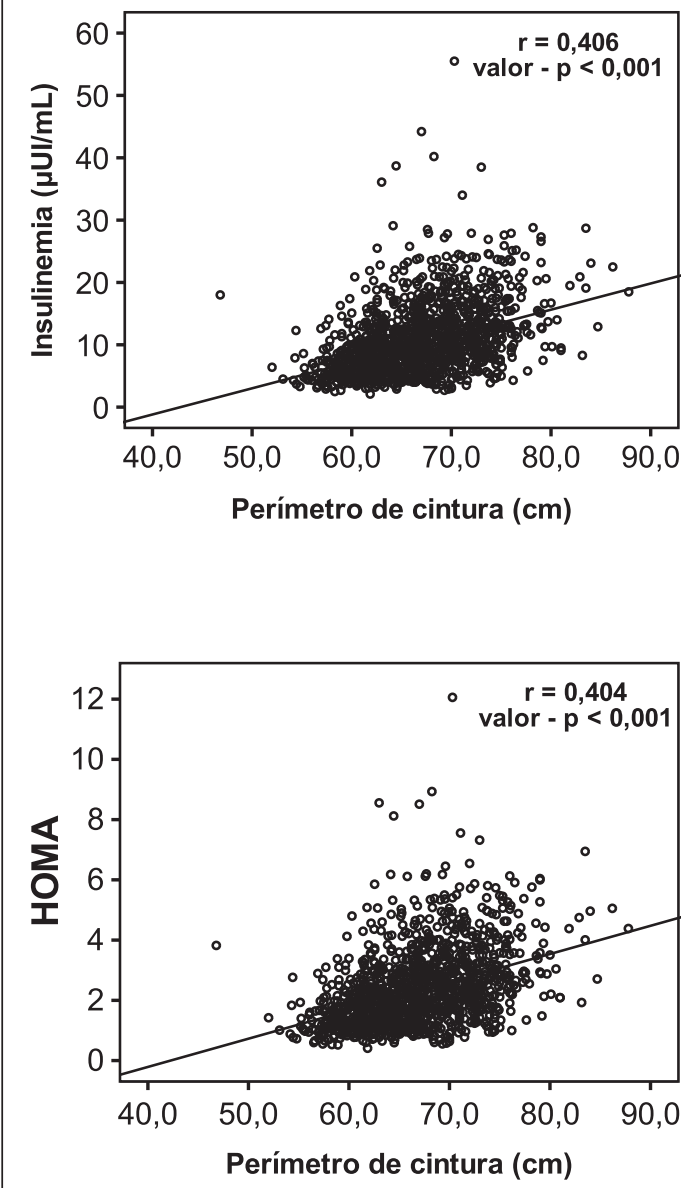

Figura 3. Correlación entre perímetro de cintura e insulinemia y HOMA en 1.192 niños eutróficos y euglicémicos. Comuna de Puente Alto, 2009-2010.
Tabla 3. Distribución percentilar de los valores de insulinemia y HOMA de acuerdo al sexo y maduración puberal, en 1.192 niños y adolescentes eutróficos y euglicémicos. Comuna de Puente Alto, 2009-2010

\begin{tabular}{|c|c|c|c|c|}
\hline \multirow[t]{3}{*}{ Percentil } & \multicolumn{4}{|c|}{ Insulinemia $(\mu \mathrm{U} / \mathrm{mL})$} \\
\hline & \multicolumn{2}{|c|}{ Mujeres } & \multicolumn{2}{|c|}{ Hombres } \\
\hline & $\begin{array}{c}\text { Tanner } \\
1+2\end{array}$ & $\begin{array}{c}\text { Tanner } \\
3+4\end{array}$ & $\begin{array}{c}\text { Tanner } \\
1+2\end{array}$ & $\begin{array}{c}\text { Tanner } \\
3+4\end{array}$ \\
\hline 99 & 25,9 & 29,1 & 22,8 & 38,7 \\
\hline 95 & 21,6 & 24,6 & 16,9 & 20,5 \\
\hline 90 & 17,8 & 22,2 & 14,0 & 17,7 \\
\hline 75 & 13,1 & 17,4 & 10,6 & 12,8 \\
\hline 50 & 9,5 & 13,2 & 7,9 & 8,9 \\
\hline 25 & 6,9 & 10,5 & 5,3 & 6,6 \\
\hline 10 & 4,9 & 7,5 & 4,2 & 5,0 \\
\hline 5 & 4,3 & 5,7 & 3,8 & 4,0 \\
\hline \multirow[t]{3}{*}{ Percentil } & \multicolumn{4}{|c|}{ HOMA } \\
\hline & \multicolumn{2}{|c|}{ Mujeres } & \multicolumn{2}{|c|}{ Hombres } \\
\hline & $\begin{array}{c}\text { Tanner } \\
1+2\end{array}$ & $\begin{array}{c}\text { Tanner } \\
3+4\end{array}$ & $\begin{array}{c}\text { Tanner } \\
1+2\end{array}$ & $\begin{array}{c}\text { Tanner } \\
3+4\end{array}$ \\
\hline 99 & 5,8 & 6,9 & 4,9 & 8,1 \\
\hline 95 & 4,9 & 5,5 & 3,9 & 4,8 \\
\hline 90 & 4,1 & 5,0 & 3,2 & 4,2 \\
\hline 75 & 2,9 & 3,8 & 2,4 & 2,9 \\
\hline 50 & 2,0 & 2,9 & 1,7 & 2,1 \\
\hline 25 & 1,5 & 2,3 & 1,2 & 1,4 \\
\hline 10 & 1,0 & 1,6 & 0,9 & 1,1 \\
\hline 5 & 0,9 & 1,2 & 0,8 & 0,9 \\
\hline
\end{tabular}




\section{Discusión}

Este estudio muestra la distribución percentilar de insulinemia y HOMA en una población pediátrica sana, eutrófica y euglicémica, destacando la influencia del sexo y maduración puberal, a la vez que su asociación al perímetro de cintura. La población que aceptó participar en el estudio llegó a $61,1 \%$, llamando la atención el sesgo por sexo ya que $68,5 \%$ de las niñas y sólo $55,5 \%$ de los varones trajeron el consentimiento firmado. Sin embargo, al restringir la muestra del estudio sólo a los eutróficos y euglicémicos, la diferencia de la composición por sexo se redujo notablemente llegando a $51,9 \%$ en niñas y $48,1 \%$ en varones.

En esta muestra las mujeres presentaron mayor insulinemia y HOMA que los hombres, dimorfismo reportado antes, aunque no siempre considerando la maduración puberal ${ }^{24-30}$. Verificamos que la diferencia por sexo es independiente de la pubertad y que el aumento de ambos parámetros con la edad depende de la pubertad, ya que en la regresión múltiple la edad no fue significativa. $\mathrm{La}$ diferencia por sexo podría deberse a diferencias en estado nutricional, composición corporal o a un desfase temporal del "estirón" de talla ${ }^{24,25}$. La primera se descarta por ser todos eutróficos y sin diferencia de zIMC según sexo, aunque sí encontramos diferencia en zT/E y en \%MG. Ésta última se estimó con fórmulas de Slaugther, método que tiene limitaciones para el uso individual, pero que presenta una adecuada correlación con el gold standard para medir MG y es recomendado para estudios poblacionales en adolescentes de ambos sexos ${ }^{31}$.

El incremento de insulinemia y de RI al progresar la pubertad ha sido bien descrito en estudios poblacionales en los Estados Unidos de Norteamérica ${ }^{14}$, así como en muestras de menor tamaño en niños chilenos obesos ${ }^{5,32}$. Ello se ha atribuido al aumento de la MG propio de este período, aunque podría más bien depender de variaciones en las señales hormonales que modulan la pubertad ${ }^{14}$.

Se ha descrito en adultos que la obesidad central o masa grasa intra-abdominal, representada por el $\mathrm{PC}$, es un factor gravitante en la asociación a $\mathrm{RI}^{33}$, lo cual se confirma en este estudio, ya que el PC tiene el primer lugar en el análisis de regresión múltiple con las variables estudiadas. Es necesario considerar que por ser una muestra eutrófica, existe menor variación del IMC y de la MG total o abdominal, a diferencia de estudios que incluyen sujetos con exceso de peso. La medición de obesidad troncal, representada por la razón o suma de pliegues cutáneos ha sido usada en estudios poblacionales ${ }^{27}$, pero presentó una débil correlación con insulinemia o RI, probablemente por su carácter indirecto y por tener menor variación en eutróficos.

La técnica para medir insulinemia es un factor importante que condiciona la heterogeneidad de los reportes; tradicionalmente se ha medido con radio-inmunoensayo (RIA), utilizando anticuerpos policlonales, siendo reemplazada posteriormente por inmunoensayos con anticuerpos monoclonales, medidos con QL, con mayor especificidad y sensibilidad ${ }^{13}$. Éstos no miden pro-insulina, péptido $\mathrm{Cu}$ otros derivados presentes en la muestra, lo cual junto al menor costo y automatización, hacen que sea la técnica más utilizada hoy en el ámbito clínico. Sin embargo, existe también diferencia entre equipos que utilizan igual técnica, como por ejemplo un estudio en Brasil reporta niveles muy inferiores en niños ${ }^{29}$. Se recomienda utilizar como referencia mediciones obtenidas con igual técnica, considerando las recomendaciones y titulación del fabricante, a la vez que realizadas en una población representativa y con estandarización adecuada ${ }^{34}$.

Para establecer puntos de corte que permitan separar valores normales de anormales, existen diferentes criterios; en adultos se definen puntos de corte para RI según el riesgo asociado de daño a la salud, con las recomendaciones generales de Brownie y Habicht ${ }^{35-37}$. En niños se ha utilizado el p75 en muestras poblacionales o p90 en muestras seleccionadas. Estos enfoques determinan normalidad según puntos de cortes definidos por la distribución percentilar, sin excluir o excluyendo, respectivamente, los casos con patologías. En nuestra muestra de 1.978 niños euglicémicos (incluyendo aquellos con exceso y déficit nutricional), con sub-grupos por sexo y edad de 250 a 700 sujetos, el p75 para insulinemia y HOMA fueron comparables al p90 de los 1.192 eutróficos aquí analizados (resultados no mostrados). En niños españoles se ha propuesto el p90:3,43 para HOMA y $15,05 \mu \mathrm{UI} / \mathrm{ml}$ para insulinemia, con valores superiores en niñas con Tanner IV-V ${ }^{28}$. Los niveles son menores en adultos: 2,6 para HOMA, validado como $+1 \mathrm{DE}$ en población chilena sana ${ }^{12}$ y como p75 en adultos mayores, asociado a RCV ${ }^{16}$.

La eutrofia es condición necesaria para determinar valores de referencia, ya que el principal 
determinante de la RI es el estado nutricional, responsable del $29 \%$ de su variación ${ }^{13,38}$. Se requiere también de representación balanceada de los estadios de maduración puberal ${ }^{39}$. En algunos estudios la definición de hiperinsulinemia o RI se ha propuesto en referencia a valores arbitrarios de sumatoria de insulinemias o análisis ROC de TTOG $^{40,41}$. Si bien los valores de P90 aquí descritos son más elevados, nos parece que constituyen un referente confiable, colaborando particularmente en su valoración en niñas púberes. El punto de corte óptimo para insulinemia y HOMA dependerá además de nuevos estudios que asocien daños a la salud en cada percentil de la distribución. Mientras éstos no estén disponibles, se propone utilizar el percentil 90, siguiendo el criterio habitual para una población sana, de acuerdo con estudios de niños antes presentados y con otro estudio chileno en adultos jóvenes sanos y eutróficos, en que se utilizó como punto de corte $1 \mathrm{DE}^{12}$. Como es esperable, los niveles son mayores que al utilizar el p75 en otros estudios en niños con diferentes estados nutricionales, punto de corte utilizado en otro estudio chileno en 800 adultos mayores, eutróficos en $20 \%$ y con expresión de diferentes factores de riesgo cardiovascular ${ }^{16}$.

Las principales fortalezas de este estudio son el buen tamaño muestral, balanceado por sexo y pubertad, el cumplimiento de eutrofia y euglicemia, la homogeneidad de las condiciones de toma de muestra y el procesamiento confiable y estandarizado para la técnica de QL. Una posible limitación es la valoración de la maduración puberal mediante auto-reporte, técnica ampliamente utilizada en estudios poblacionales, siendo la concordancia entre el personal de salud y el auto-reporte adecuada en ambos sexos, aunque mejor en las niñas ${ }^{42}$. Estudios recientes en población china apoyan el uso del auto-reporte ${ }^{43,44}$, sin cuestionarse su uso en estudios poblacionales, mientras que para estudios clínicos o individuales se requiere complementar con evaluación especializada. Otra limitación es la falta de representación de edades $<10$ años y de post-púberes.

En conclusión, se entrega una referencia local, con la cual no se contaba, que puede ayudar a orientar la valoración clínica de insulinemia y HOMA. Se está trabajando en identificar marcadores de daño que permitan justificar el punto de corte sugerido, en especial considerando su relación a PC.

\section{Referencias}

1. Martin BC, Warram JH, Krolewski AS, Bergman RN, Soeldner JS, Kahn CR. Role of glucose and insulin resistance in development of type 2 diabetes mellitus: results of a 25-year follow-up study. Lancet 1992; 340 (8825): 925-9.

2. Reaven GM. Banting lecture 1988. Role of insulin resistance in human disease. Diabetes 1988; 37: 1595-607.

3. Cook S, Weitzman M, Auinger P, Nguyen M, Dietz WH. Prevalence of a metabolic syndrome phenotype in adolescents: findings from the third National Health and Nutrition Examination Survey, 1988-1994. Arch Pediatr Adolesc Med 2003; 157: 821-7.

4. Freedman DS, Dietz WH, Srinivasan SR, Berenson GS. The relation of overweight to cardiovascular risk factors among children and adolescents: the Bogalusa Heart Study. Pediatrics 1999; 103: 1175-82.

5. Barja S, Arteaga A, Acosta AM, Hodgson MI: Resistencia insulínica y otras expresiones del Síndrome Metabólico en niños obesos chilenos. Rev Med Chile 2003; 131: 25968.

6. Barja S, Arnaíz P, Acevedo M, Berríos X, Guzmán B, Bambs C, et al. Marcadores de aterosclerosis precoz y Síndrome Metabólico en niños. Rev Med Chile 2009; 137: 522-30.

7. Weiss R, Caprio S, Trombetta M, Taksali SE, Tamborlane WV, Bonadonna R. Beta-cell function across the spectrum of glucose tolerance in obese youth. Diabetes 2005; 54: 1735-43.

8. Matthews DR, Hosker JP, Rudenski AS, Naylor BA, Treacher DF, Turner RC. Homeostasis model assessment: insulin resistance and beta-cell function from fasting plasma glucose and insulin concentrations in man. Diabetología 1985; 28: 412-9.

9. Bonora E, Targher G, Alberiche M, Bonadonna RC, Saggiani F, Zenere MB, et al. Homeostasis model assessment closely mirrors the glucose clamp technique in the assessment of insulin sensitivity: studies in subjects with various degrees of glucose tolerance and insulin sensitivity. Diabetes Care 2000; 23: 57-63.

10. Gungor N, Saad R, Janosky J, Arslanian S. Validation of surrogate estimates of insulin sensitivity and insulin secretion in children and adolescents. J Pediatr 2004; 144: 47-55.

11. Eschwege E, Richard JL, Thibult N, Ducimetiere P, Warnet JM, Claude JR, et al. Coronary heart disease mortality in relation with diabetes, blood glucose and plasma insulin levels. The Paris prospective study, ten years later. Horm Metab Res Suppl 1985; 15: 41-6.

12. Acosta AM, Escalona M, Maiz A, Pollak F, Leighton 
F. Determinación del índice de resistencia insulínica mediante HOMA en una población de la Región Metropolitana de Chile. Rev Med Chile 2002; 130: 1227-31.

13. Chevenne D, Trivin F, Porquet D. Insulin assays and reference values. Diabetes \& Metabolism 1999; 25: 459-76.

14. Moran A, Jacobs DR Jr, Steinberger J, Hong CP, Prineas $\mathrm{R}$, Luepker $\mathrm{R}$, et al. Insulin resistance during puberty: results from clamp studies in 357 children. Diabetes 1999; 48: 2039-44.

15. Burrows AR, Leiva BL, Burgueño AM, Maggi MA, Giadrosic RV, Díaz B, et al. [Insulin sensitivity in children aged 6 to 16 years: association with nutritional status and pubertal development]. Rev Med Chile 2006; 134: 1417-26.

16. Garmendia ML, Lera L, Sánchez H, Uauy R, Albala C. [Homeostasis model assessment (HOMA) values in Chilean elderly subjects]. Rev Med Chile 2009; 137: 1409-16.

17. NHANES-2000 CDC Clinical Growth Charts for the United States. Disponible en: http://www.cdc.gov/nccdphp/dnpa/growthcharts/training/modules/module2/ text/page6b.htm (Último acceso: 29 de julio 2011).

18. NHANES - Anthropometry Procedures Manual, January 2004. Disponible en: http://www.cdc.gov/nchs/data/ nhanes/nhanes_03_04/BM.pdf (Último acceso: 18 de enero 2011).

19. Fernández J, Redden D, Pietrobelli A, Allison D. Waist circumference percentiles in nationally representative samples of African-American, European-American and Mexican-American children and adolescents. J Pediatr 2004; 145: 439-44.

20. Slaughter M, Lohman T, Boileau R, et al. Skinfold equations for estimation of body fatness in children and youth. Hum Biol 1988; 60: 709-23.

21. Urrejola P, Hodgson M, Icaza M. Evaluación de la composición corporal en niñas usando impedanciometría bioeléctrica y pliegues subcutáneos. Rev Chil Pediatr 2001; 72: 26-33.

22. Liberona Y, Castillo O, Engler V, Villarroel L, Rozowski J. Nutritional profile of schoolchildren from different socio-economic levels in Santiago, Chile. Public Health Nutr 2011; 14: 142-9.

23. Tanner JM. (1962). Growth at adolescence ( $2^{\text {nd }}$ Ed.). Oxford: Blackwell Scientific Publications.

24. Guillaume M, Lapidus L, Beckers F, Lambert A, Björntorp P. Cardiovascular risk factors in children from the Belgian province of Luxembourg. The Belgian Luxembourg Child Study. Am J Epidemiol 1996; 144: 867-80.

25. Allard P, Delvin E, Paradis G, Hanley J, O'Loughlin J, Lavallée C, et al. Distribution of fasting plasma insulin, free fatty acids, and glucose concentrations and of homeostasis model assessment of insulin resistance sample of Quebec children and adolescents. Clinical Chemistry 2003; 49: 644-9.

26. Francois A, Maumus S, Vicent-Viry M, Gueguen R, Siest G, Visvikis S. Age and sex-related reference values for serum insulin concentration and its biological determinants in a French healthy population. The STANISLAS cohort. Clin Chem Lab Med 2004; 42: 1140-9.

27. Misra A, Vikram NK, Arya S, Pandey RM, Dhingra V, Chatterjee A, et al. High prevalence of insulin resistance in post pubertal Asian Indian children is associated with adverse truncal body fat patterning, abdominal adiposity and excess body fat. Int J Obes Relat Metab Disord 2004; 28: 1217-26.

28. García Cuartero B, García Lacalle C, Jiménez Lobo C, González Vergaz A, Calvo Rey C, Alcázar Villar MJ, et al. Índice HOMA y QUICKI, insulina y péptido C en niños sanos. Puntos de corte de riesgo cardiovascular. An Pediatr (Barc) 2007; 66: 481-90.

29. Almeida C, Pinho A, Ricco R, Pepato MT, Brunetti I. Determination of glycemia and insulinemia and HOMA in schoolchildren and adolescents with normal body mass index. J Pediatr (Rio J) 2008; 84: 136-40.

30. Denney-Wilson E, Cowell CT, Okely AD, Hardy LL, Aitken R, Dobbins T. Associations between insulin and glucose concentrations and anthropometric measures of fat mass in Australian adolescents. BMC Pediatr 2010; 10: 58.

31. Rodríguez G, Moreno LA, Blay MG, Blay VA, Fleta J, Sarría A, et al. AVENA-Zaragoza Study Group. Body fat measurement in adolescents: comparison of skinfold thickness equations with dual-energy X-ray absorptiometry. Eur J Clin Nutr 2005; 59: 1158-66.

32. Burrows R, Burgueño M, Leiva L, Ceballos X, Guillier I, Gattas V, et al. [Cardiovascular risk and metabolic profile in obese children and adolescents with low insulin sensitivity]. Rev Med Chile 2005; 133: 795-804.

33. Grundy SM, Cleeman JI, Daniels SR, Donato KA, Eckel RH, Franklin BA, et al. Diagnosis and management of the metabolic syndrome: an American Heart Association/National Heart, Lung, and Blood Institute Scientific Statement. Circulation 2005; 112: 2735-51.

34. Staten MA, Stern MP, Miller WG, Steffes MW, Campbell SE. Insulin Standardization Workgroup. Insulin assay standardization: leading to measures of insulin sensitivity and secretion for practical clinical care. Diabetes Care 2010; 33: 205-6.

35. Rutter MK, Wilson PW, Sullivan LM, Fox CS, D’Agostino RB Sr, Meigs JB. Use of alternative thresholds defining insulin resistance to predict incident type 2 diabetes mellitus and cardiovascular disease. Circulation 2008; 117: 1003-9.

36. Brownie C, Habicht JP. Selecting a screening cut-off 
Insulinemia y HOMA en niños y adolescentes chilenos - S. Barja et al

point or diagnostic criterion for comparing prevalence of disease. Biometrics 1984; 40: 675-84.

37. Brownie C, Habicht JP, Cogill B. Comparing indicators of health or nutritional status. Am J Epidemiol 1986; 124: 1031-44.

38. Lee JM, Okumura MJ, Davis MM, Herman WH, Gurney JG. Prevalence and determinants of insulin resistance among U.S. adolescents: a population-based study. Diabetes Care 2006; 29: 2427-32.

39. Morales LM, Raleigh X, Fernández V, Molero-Conejo E. Distribución de las concentraciones de glucosa e insulina basal, HOMA IR Y HOMA ßcell en niños y adolescentes de la ciudad de Maracaibo, Venezuela. Rev Med Chile 2007; 135: 205-11.

40. Keskin M, Kurtoglu S, Kendirci M, Atabek ME, Yazici C. Homeostasis model assessment is more reliable than the fasting glucose/insulin ratio and quantitative insulin sensitivity check index for assessing insulin resistance among obese children and adolescents. Pediatrics 2005; 115: e500-3.

41. Atabek ME, Pirgon O. Assessment of insulin sensitivity from measurements in fasting state and during an oral glucose tolerance test in obese children. J Pediatr Endocrinol Metab 2007;20:187-95.

42. Coleman L, Coleman J. The measurement of puberty: a review. Journal of Adolescence 2002; 25: 535-50.

43. Chan NP, Sung RY, Kong AP, Goggins WB, So HK, Nelson EA. Reliability of pubertal self-assesment in Hong Kong Chinese Children. J Paediatr Child Health 2008; 44: 353-8.

44. Chan NP, Sung RY, Nelson EA, So HK, Tse YK, Kong AP. Measurement of pubertal status with a Chinese self-reported pubertal development scale. Matern Child Health J 2010; 14: 466-73. 